PAPER

\title{
Progression of structural neuropathology in preclinical Huntington's disease: a tensor based morphometry study
}

\author{
C M Kipps, A J Duggins, N Mahant, L Gomes, J Ashburner, E A McCusker
}

See Editorial Commentary, p 620

J Neurol Neurosurg Psychiatry 2005;76:650-655. doi: 10.1136/jnnp.2004.047993

\begin{abstract}
See end of article for authors' affiliations

Correspondence to: Dr C M Kipps, Neurology Unit, University of Cambridge, R3 Neurosciences, Addenbrooke's Hospital, Cambridge CB2 2QQ, UK; cmk41@medschl.cam. ac.uk
\end{abstract}

Received 18 June 2004 Revised version received 30 September 2004 Accepted 1 October 2004
Background and objectives: Regional cerebral atrophy occurs in carriers of the Huntington's disease (HD) gene mutation before clinical diagnosis is possible. The current inability to reliably measure progression of pathology in this preclinical phase impedes development of therapies to delay clinical onset. We hypothesised that longitudinal statistical imaging would detect progression of structural pathology in preclinical carriers of the HD gene mutation, in the absence of measurable clinical change.

Methods: Thirty subjects (17 preclinical mutation positive, 13 mutation negative) underwent serial clinical and magnetic resonance imaging (MRI) assessments over an interval of 2 years. Statistically significant changes in regional grey and white matter volume on MRI were analysed using tensor based morphometry (TBM). This technique derives a voxel-wise estimation of regional tissue volume change from the deformation field required to warp a subject's early to late T1 images.

Results: Over 2 years, there was progressive regional grey matter atrophy in mutation-positive relative to negative subjects, without significant clinical progression of disease. Significant grey matter volume loss was limited to bilateral putamen and globus pallidus externa (GPe), left caudate nucleus, and left ventral midbrain in the region of the substantia nigra.

Conclusions: While these results are consistent with previous cross sectional pathologic and morphometric studies, significant progression of atrophy in HD before the onset of significant clinical decline is now demonstrable with longitudinal statistical imaging. Such measures could be used to assess the efficacy of potential disease modifying drugs in slowing the progression of pathology before confirmed clinical onset of $\mathrm{HD}$.
$\mathrm{N}$ eurodegenerative diseases are characterised by gradual progression of neuropathology. ${ }^{1}$ While clinical signs may also accumulate over time, it is only when these exceed the limits of normal variation that clinical diagnosis is possible. In Huntington's disease (HD) clinical diagnosis is usually delayed until middle age. ${ }^{2}$ In contrast, identification of the relevant CAG triplet repeat expansion within the huntingtin gene allows definitive genetic diagnosis of HD in utero, although predictive genetic testing is usually delayed until the age of consent. ${ }^{3-5}$

The availability of genetic testing has afforded an opportunity to study the natural history of HD prior to clinical diagnosis. Conceivably, the delay in clinical diagnosis may relate to the insensitivity of the clinical instruments employed in detecting subtle but real decline in function. Alternatively, perhaps there is true functional reserve in those brain structures affected by the HD pathologic process.

In support of the first possibility, several studies using careful clinical assessment or standardised clinical rating scales have identified statistically significant abnormalities in cohorts of mutation-positive subjects who individually would not meet established clinical criteria for definite HD. ${ }^{67}$ Similarly, repeated clinical measurements within such mutation-positive "preclinical" subjects over intervals of only 2 years demonstrate significant progression prior to clinical diagnosis. ${ }^{8}$ Even in individuals, more refined motor or cognitive testing directed at postulated pathophysiology and regional cerebral dysfunction can bring forward clinical diagnosis. ${ }^{9-11}$ However, it is difficult to explain characteristic pathologic changes found at post mortem in young mutationpositive individuals long before anticipated clinical onset. ${ }^{12}{ }^{13}$ Moreover, using statistical imaging techniques, significant regional cerebral atrophy in a mutation-positive preclinical cohort exceeds that which might explain subtle clinical changes. ${ }^{14}$ These latter findings imply that there is a prolonged phase of subclinical pathologic progression that precedes any functional decline, and raise the possibility that an intervention effective in slowing pathologic progression might extend the period during which the mutation-positive individual lives without significant morbidity from HD.

Several potential neuroprotective agents have been suggested for evaluation in preclinical HD, including creatine, ${ }^{15}$ minocycline, ${ }^{16}$ and coenzyme Q10. ${ }^{17}$ The ideal time for such an intervention might depend on what stage of $\mathrm{HD}$ pathophysiology the agent is postulated to interrupt, but if subclinical pathologic progression is indeed prolonged this is likely to be early in the life of the mutation-positive individual, prior to the development of neurological dysfunction.

The motivation for the current study was to identify significant structural progression in the absence of significant clinical progression in an adult mutation-positive preclinical cohort relative to mutation negatives over a 2 year interval. We chose to investigate progressive regional cerebral atrophy using statistical imaging as a marker of progression. We use the term "statistical imaging" to encompass the spectrum of computational neuroanatomic techniques in which voxel based statistics quantify regional changes in cerebral tissue volume, including voxel based morphometry (VBM) and tensor based morphometry (TBM). Previous work by our group and others has demonstrated that VBM is a sensitive indicator of preclinical structural pathology in both early and preclinical HD. ${ }^{14} 18$

Abbreviations: GM segment, grey matter segment; GPe, globus pallidus externa; HD, Huntington's disease; MNI, Montreal Neurologic Institute; $\mathrm{MRI}$, magnetic resonance imaging; $\mathrm{SNr}$, substantia nigra pars reticulata; SVC, small volume correction; TBM, tensor based morphometry; UHDRS, United Huntington's Disease Rating Scale; VBM, voxel based morphometry; WM segment, white matter segment 
Pathologically, the striatum and its projection fibres are disproportionately affected by the disease. The caudate nucleus and the dorsal putamen are believed to be the earliest sites of pathology, as are the targets of striatal projection fibres such as the globus pallidus externa (GPe) and substantia nigra pars reticulata (SNr). ${ }^{12}{ }^{19-24}$ Atrophy of these regions correlates well with clinical measures of disease severity, ${ }^{19}$ and with loss of GABA containing medium spiny neurons. ${ }^{25}$ Other work has highlighted prominent and widespread cortical and white matter loss. ${ }^{12} 222627$

Atrophy of the head of the caudate with ventricular dilatation has long been recognised as a cardinal sign of established HD on imaging. In preclinical disease, however, studies using manual morphometric techniques identify the putamen as having the earliest volume reduction. ${ }^{28} 29$ There are relatively few longitudinal studies assessing progressive volume changes in basal ganglia measurements, and, until recently, these have only reported volume changes in the caudate nucleus. ${ }^{30-32}$ It is unclear, however, whether manual morphometry yielded any additional information over thorough clinical assessment, and the rate of structural change did not correlate with that measured using clinical rating scales. Moreover, conventional morphometric techniques, which for practical reasons must be limited to a small number of structures, inevitably ignore the possibility that more widespread changes are present in preclinical disease. The validity of such manual demarcation is dependent on the skill of the observer and inter-observer reliability. In contrast, the results of statistical imaging techniques are unbiased and objective, and thus potentially reproducible by different operators. Using statistical imaging, regional prior hypotheses can be accommodated in a principled way by limiting the correction for multiple comparisons to voxels within a small volume of interest. Based on the weight of evidence presented above, our regional prior hypothesis of progressive grey matter atrophy was limited to basal ganglia structures. Importantly though, whole brain statistical imaging techniques also allow identification of significant atrophy in regions not predicted by prior hypothesis if such volume change survives whole brain correction.

\section{METHODS \\ Subjects}

The Western Sydney Area Health Service Institutional Ethics Committee approved the protocol. All subjects gave written informed consent. The subject and control groups were recruited from a population who had undergone predictive genetic testing through the New South Wales (Australia) genetics service. All predictive tests were preceded by a neurological assessment by a specialist neurologist (EM) who has extensive experience in the assessment of patients with HD, and who had concluded that there was no clinical evidence of the disorder. Full details of the group have been reported previously. ${ }^{14}$

All subjects were followed up, and invited to undergo reassessment and magnetic resonance imaging (MRI) scanning approximately 2 years after their first involvement. Of 34 subjects recruited initially (18 mutation positive, 16 mutation negative), 30 subjects were willing to be involved in a subsequent assessment. Three subjects (mutation negative) from the original cohort declined further evaluation, and one subject (mutation positive) was unable to be re-scanned because a new medical condition precluded this. Demographic characteristics of the cohort are given in table 1. There were no significant differences between mutation-positive and mutation-negative subjects with respect to age, gender, or the time interval between scans.
Table 1 Demographic characteristics by gene status

\begin{tabular}{llll}
\hline & $\begin{array}{l}\text { HD mutation } \\
\text { negative }\end{array}$ & $\begin{array}{l}\text { HD mutation } \\
\text { positive }\end{array}$ & All subjects \\
\hline Age & $42.0(11.4)$ & $43.8(10.0)$ & $43.0(10.5)$ \\
Sex (M/F) & $9 / 4$ & $7 / 10$ & $16 / 14$ \\
Interval (months) & $22.8(2.1)$ & $21.9(2.0)$ & $22.3(2.0)$ \\
Handed (R/L/B) & $9 / 2 / 2$ & $11 / 4 / 2$ & $20 / 6 / 4$ \\
CAG repeats & $20(3.3)$ & $41(2.8)$ & - \\
\hline
\end{tabular}

Values are mean (standard deviation). There were no significant between group differences in age, gender, or time interval between scans.

\section{Clinical assessment}

Each subject underwent a second formal motor and cognitive examination by a neurologist experienced in the clinical assessment of HD who remained blinded to genetic status (CK). A different rater had performed identical testing in the first phase of the study, but after the first phase analysis was no longer blinded to genetic status and thus could not be involved in follow up assessments. On each assessment, subjects were scored according to the motor component of the United Huntington's Disease Rating Scale (UHDRS), which quantifies chorea, bradykinesia, rigidity, motor impersistence, motor sequencing, ocular movements, and gait. Scores range from 0 to 128 , with higher scores representing greater motor impairment. The scale has been validated, and when applied to mutation-positive subjects with established disease, it is internally consistent, has inter-observer reliability, and is useful for tracking disease progression. ${ }^{33}$ Videotaped assessments were viewed by the other two study neurologists (EM, NM) in order to assess whether any subjects had progressed to the point where confident clinical diagnosis of HD was possible.

On each assessment, the cognitive component of the UHDRS (phonetic verbal fluency test, symbol digit modalities test, Stroop word, Stroop colour, and Stroop colour-word test) was also administered.

Each clinical score was analysed using a general linear model, in which age and years of formal education were included as nuisance covariates. Deviation from the null hypothesis of no effect of gene status on clinical progression was measured by comparing the late minus early difference in score between mutation-positive and negative subjects, with significance quantified as a paired $t$ statistic.

\section{Imaging}

Early and late whole brain structural MRI was performed using the same high resolution 3D Tl weighted MP_RAGE sequence on the same $1.5 \mathrm{~T}$ scanner in all subjects with the following parameters: TR/TE (echo time): 9.7/4, flip angle $15^{\circ}$, matrix $256 \times 256$, FOV (field of view) $250 \mathrm{~mm}$. Slices ( $1 \mathrm{~mm}$ ) were acquired in the coronal plane. Between early and late phases of the study there had been no significant upgrade or modification of the scanner. All stages of image preprocessing and statistical analysis were performed using freely available software (spm99, spm2b, The Wellcome Department of Imaging Neuroscience, London; http://www.fil.ion.ucl.ac.uk/ spm) on a Matlab 6.1 platform (MathWorks, Natick, MA, USA).

TBM was used to compare, between gene status groups, changes over time in regional tissue volume. In this technique, a deformation field characterises the high dimensional warp required to approximate early to late Tl images within subject. The following procedure, illustrated in the supplementary material (available at http://jnnp.bmjjournals. com/supplemental/), was applied to the scans of each subject: 
1. Early and late Tl images were first manually re-oriented to place the anterior commissure at the origin of the three-dimensional Montreal Neurologic Institute (MNI) coordinate system. A rigid registration of early to late images was performed, by maximising the mutual information of the joint intensity histogram of the images.

2. We sought a high dimensional deformation field (using the spm2b "Deformation Toolbox") that would warp the early $\mathrm{Tl}$ image to match the late $\mathrm{Tl}$ image within subject. ${ }^{34}$ This approach is based on minimising the mean squared difference between the images. A regularisation term was also included in the objective function, which kept the deformations smooth, and enforced a one-toone mapping. The trade off between the mean squared difference between the images and the smoothness of the deformations was defined by a regularisation parameter, which we set to four. Eight iterations of the algorithm were considered sufficient to model the deformations that were likely to occur within subject over time.

All of the steps described above were performed using spm $2 \mathrm{~b}$ software; all steps to be described below were performed using spm99.

3. The result of the high dimensional registration (step 2) is a detailed deformation field that contains a mapping from each point in the late image, to the corresponding point in the early image, for each subject. The amount of regional expansion or contraction was extracted from this field, by taking the determinant of the gradient of the deformation at each point (Jacobian determinant). ${ }^{35}$ The map of Jacobian determinants is in alignment with the late image, and encodes the number of $\mathrm{mm}^{3}$ in the early image that match to $1 \mathrm{~mm}^{3}$ in the late image. For example, if some structure uniformly contracted from $1500 \mathrm{~mm}^{3}$ down to $1000 \mathrm{~mm}^{3}$, then the value of the Jacobian determinant map would contain a value of 1.5 in regions corresponding to that structure. The early Tl images were not used again in subsequent preprocessing and analysis.

4. The late image was segmented using spm99. ${ }^{36}$ This produces maps of the probability of each voxel in the late image being grey matter (GM segment) and white matter (WM segment). Values are in the range of zero and one, where larger values indicate a higher tissue probability. The sum of voxel values in any region of the GM segment indicates the amount of grey matter in that region of the late image.

5. To compare regional volume change between groups it was necessary to spatially normalise images to a common stereotactic space to ensure that the same voxel in different subjects sampled an approximately corresponding neuroanatomic structure. Spatial normalisation parameters for each subject were estimated by warping the late GM segment (from step 4) to best match an unbiased grey matter template. Normalisation involved a 12 parameter affine transformation followed by a non-linear deformation consisting of a linear combination of $(7 \times 8 \times 7)$ low frequency periodic basis functions. A customised template was created by averaging smoothed ( $8 \mathrm{~mm}$ isotropic Gaussian kernel), normalised, late GM segments from all subjects, in this case registered to the published MNI Tl image.

6. The estimated spatial normalisation parameters (from step 5) were applied to the GM and WM segments (from step 4), and also to the Jacobian determinants (from step 3). Similar to the high dimensional warping step, the spatial normalisation step also results in regional volume changes.

7. A measure of tissue specific volume change was derived from the estimated high dimensional warp. For each subject, the normalised GM and WM segments (from step 6) are multiplied, voxel-by-voxel, by the Jacobian determinant map to form a product image. It is assumed that the early image warped to match the late image would produce the same segmentation result as that obtained by segmenting the late image. This step produces images that represent the amount of grey and white matter in each region of the early image, but in a form that is in alignment with the late image. Clearly voxel values in the product image would exceed corresponding values in the late tissue segment where regional tissue contraction had occurred. Errors of segmentation by which extra-cerebral tissue was misclassified as grey matter (for example) were eliminated by multiplication with a subject specific binary inclusive mask identifying brain tissue.

8. Where normalisation contracts grey matter to approximate the template, local grey matter concentration will be underestimated (similarly expansion will cause overestimation). To preserve the original tissue volumes, the normalised tissue class and normalised product images were modulated by the Jacobian determinants from the spatial normalisation (note that these are not the determinants from the high dimensional warping of early to late image). These modulated images were then smoothed with a $12 \mathrm{~mm}$ isotropic Gaussian kernel to accommodate inexact spatial normalisation.

The general linear model was used for statistical inference. A design matrix was constructed in which four condition columns of ones and zeros identified data in rows from mutation-negative product images, mutation-negative tissue segments, mutation-positive products, and mutation-positive segments, respectively. Thirty, subject specific, dummy covariates modelled the variance attributable to repeated measures within subject. Age and sex nuisance covariates modelled potential confounding effects on regional cerebral atrophy. Inference was based on a $[-1,1,1,-1]$ contrast of parameter estimates for each voxel, using two tailed $t$ statistics. In effect, we performed a paired $t$ test to determine whether greater tissue contraction had occurred in the mutation-positive relative to mutation-negative subjects. Where a regional prior hypothesis existed, $\mathrm{p}$ values were corrected for multiple comparisons across regions of interest (table 2), by using an appropriate small volume correction (SVC). Elsewhere, whole brain correction was used to determine significance. Separate grey and white matter analyses were performed.

\section{RESULTS}

A VBM analysis of the distribution of grey matter atrophy attributable to HD gene status on initial MRI has been published elsewhere. ${ }^{14}$ An identical analysis of the second MRI on the slightly reduced number of subjects scanned in the second phase of the study revealed a qualitatively similar distribution of atrophy.

TBM identified significant grey matter volume loss over time in mutation positives relative to mutation negatives limited to the basal ganglia (table 2, figs 1 and 2). On the left, this involved the medial putamen, lateral GPe, and lateral head of caudate including the striatal bridges extending across the anterior limb of the internal capsule. There was a separate area of significant grey matter atrophy at a location in the ventral midbrain consistent with the left substantia nigra. There was symmetric but slightly less significant 
Table 2 Regional volume contraction

\begin{tabular}{|c|c|c|c|c|c|c|c|}
\hline \multirow[b]{2}{*}{ Segment } & \multirow[b]{2}{*}{ Change } & \multicolumn{3}{|c|}{ MNI coordinates } & \multirow[b]{2}{*}{ z Scores } & \multirow{2}{*}{$\begin{array}{l}\text { p Corrected } \\
(\text { SVC, } \mathrm{mm})^{*}\end{array}$} & \multirow[b]{2}{*}{ Region } \\
\hline & & $\mathbf{x}$ & $y$ & $\mathbf{z}$ & & & \\
\hline Grey & Contraction & -21 & -4.5 & 1.5 & 3.69 & $0.004(10)$ & Left putamen \\
\hline Grey & Contraction & 19.5 & 0 & 1.5 & 3.01 & $0.03(10)$ & Right putamen and GPe \\
\hline Grey & Contraction & -4.5 & -21 & -16.5 & 3.11 & $0.006(5)$ & Left (ventral) midbrain \\
\hline
\end{tabular}

*The small volume correction (SVC) indicated is the radius of a sphere in $\mathrm{mm}$.

Regional volume contraction of mutation-positive relative to control subjects (local maxima).

putaminal and pallidal atrophy on the right, although right caudate and nigral atrophy did not reach significance. There was no significant effect of age or trinucleotide repeat length on the rate of atrophy. No significant interval change in regional white matter volume could be identified.

An analysis of clinical scores attributable to HD gene status at initial assessment has been published elsewhere. ${ }^{14}$ An identical analysis of clinical scores on the 30 subjects remaining at follow up was qualitatively similar, revealing a trend towards higher UHDRS motor and lower cognitive scores in mutation-positive subjects. Verbal fluency and Stroop word scores were each significantly lower in mutation-positive subjects (verbal fluency $\mathrm{p}<0.048$; Stroop word $\mathrm{p}<0.010)$.

In contrast, there was no significant decline in motor or cognitive function attributable to HD gene status in the interval between assessments. Indeed changes in scores on serial assessment did not even show a consistent trend toward clinical deterioration (fig 3). No subject was felt to have progressed to the point of confirmed clinical diagnosis by all three raters on review of videotaped assessments, although one rater (EM) felt this was the case with one mutation-positive subject.

\section{DISCUSSION}

We have shown progressive regional grey matter atrophy on serial MRI in preclinical HD, despite a lack of clinically detectable onset or progression of disease in the same group. This is the first study to show longitudinal structural changes in the putamen, GPe, and ventral midbrain in preclinical subjects, over such a short time interval.

The progressive atrophy demonstrated in the putamen was medial and dorsal, extending laterally, consistent with the predictions of neuropathological studies by Vonsattel and others. ${ }^{19}{ }^{24}$ Hedreen and Folstein ${ }^{37}$ showed loss of medium spiny neurons throughout the dorso-ventral extent of the
A

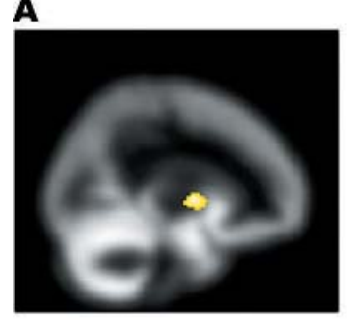

D

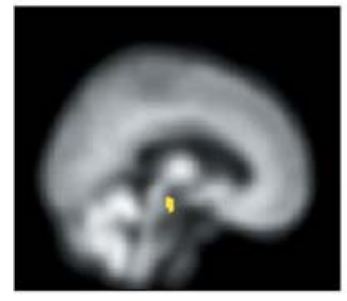

G

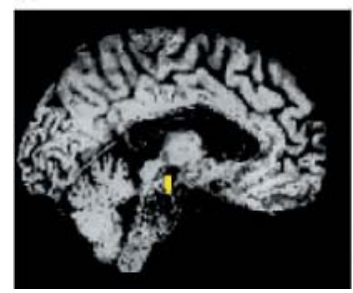

B

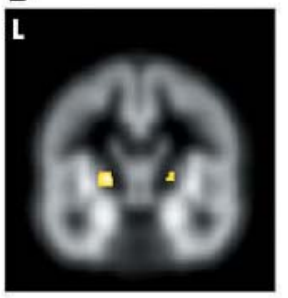

$\mathbf{E}$

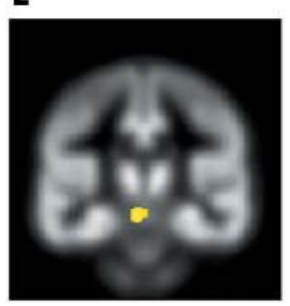

H

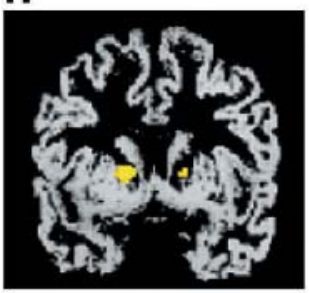

C

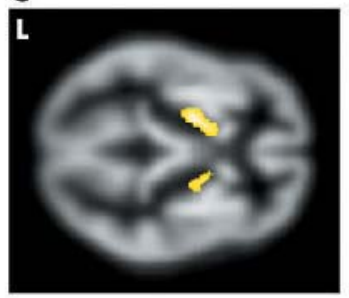

$\mathbf{F}$

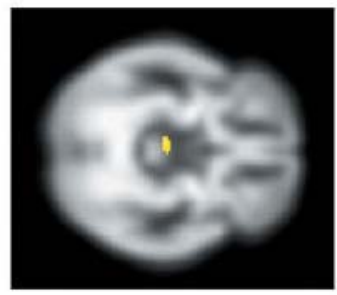

I

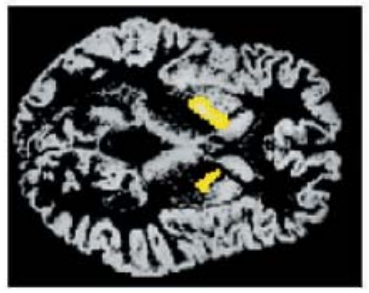

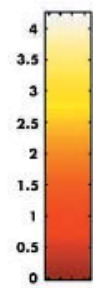

Figure 1 Statistical parametric map of main effect of HD gene status on local grey matter volume change over time. This contrast identifies areas of interval grey matter contraction in mutation-positive relative to mutation-negative subjects. The threshold for display is $p<0.005$ (uncorrected). $z$ Scores are indicated by colour temperature according to the scale. (A-F) Results rendered on the mean of late normalised GM segments from all 30 subjects. $\mathrm{MNI}$ coordinates $(\mathrm{mm})$ of sections $A-C[x=-21 y=-4.5 z=1.5]$ and $D-F[x=-4.5 y=-21 z=-16.5]$. (G-1) Results rendered on a late normalised GM segment of one mutation-negative subject. MNI coordinates $(\mathrm{mm})$ of sections $\mathrm{G}-\mathrm{I}[\mathrm{x}=-2.5 \mathrm{y}=1.5 \mathrm{z}=6.0]$. 

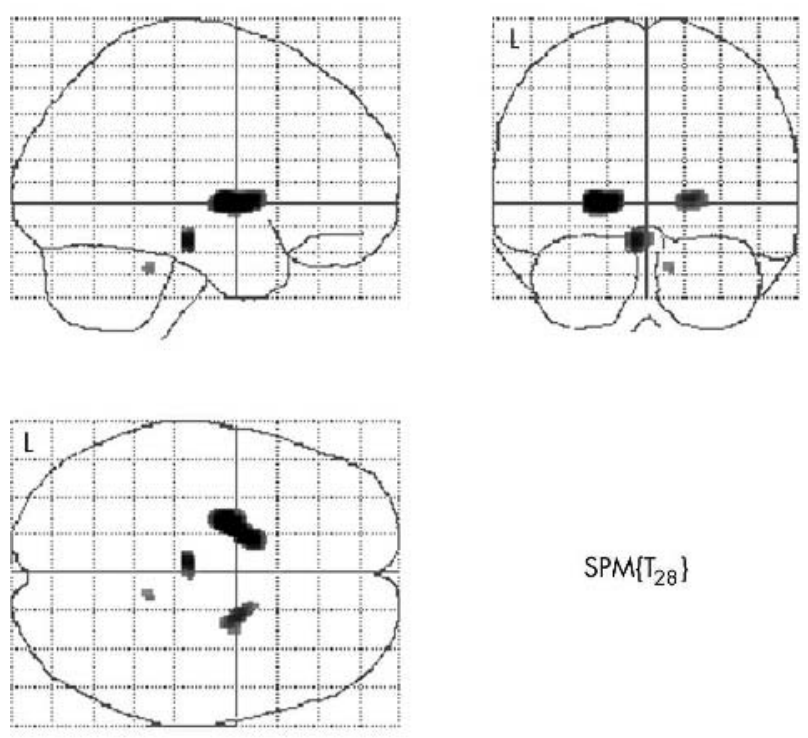

$\operatorname{SPM}\left\{\mathrm{T}_{28}\right\}$

Height threshold $\mathrm{T}=2.76$

Extent threshold $k=0$ voxels

Figure 2 "Glass brain" projections of the main effect of HD gene status on local grey matter volume change over time.

caudate and putamen as an early pathological change in HD. Marked atrophy of GPe has long been noted in pathological studies of early and preclinical HD, and is believed to be, in part, secondary to loss of striatal projection fibres. ${ }^{12} 202138$ This contrasts with a relative preservation of the globus pallidus interna early in the disease, again a finding of our study.

This distribution of progressive grey matter atrophy over 2 years is consistent with the conclusions of Aylward and coworkers from a cross sectional study, and a recent longitudinal manual morphometry study, of subjects approaching predicted clinical onset. ${ }^{39}$ From these studies it is apparent that both the putamen and the caudate are predominant early sites of pathology, and that the rate of putaminal atrophy exceeds that of the caudate nucleus in subjects furthest from expected clinical onset. By choosing a preclinical cohort on the basis of greater repeat numbers and younger age of parental onset, and by rescanning subjects after a longer interval than was employed in our work, these investigators presumably selected subjects who had accumulated a greater burden of pathology than was present in our unselected mutation-positive preclinical cohort. Their findings confirm our results using an alternate method, but also highlight the greater sensitivity of TBM over manual morphometric techniques. Furthermore, our use of a longitudinal control group minimises the possibility that age related volume loss was the explanation for the basal ganglia atrophy.

The progressive left sided midbrain atrophy noted in our study would appear to be in the region of the substantia nigra. Substance P-containing projection fibres to the $\mathrm{SNr}$ from the striatum degenerate preferentially in $\mathrm{HD}^{21}$ Furthermore, the SNr influences initiation of saccadic eye movements, one of the earliest abnormalities in $\mathrm{HD}^{40}$ Although such converging lines of evidence support this localisation, anatomical precision in this region is limited by the effects of the Gaussian smoothing process, and the relative size of the $\mathrm{SNr}$ itself.

The suggestion of a possible left sided asymmetry in the distribution of atrophy is intriguing, and is consistent with our previous work ${ }^{14}$ and that of Rosas et $a l^{41}$ in a study of cortical ribbon thickness in HD. It has been postulated that increased lactate levels in the left striatum reflect increased input from the dominant hemisphere, and greater excitotoxic stress to these GABAergic neurons and their projections. ${ }^{42}$

We did not see progressive cortical or white matter atrophy, despite our original findings of early cortical involvement. ${ }^{14}$ Could it be that cortical volume loss in the mutation-positive subjects occurred very early in life and was complete before the first MRI? This seems unlikely given that significant and widespread cortical atrophy is a feature of end stage disease..$^{23}$ Perhaps atrophy of cortical and white matter structures progresses at a different rate from that of deep grey matter, ${ }^{26}$ with change over a 2 year interval being negligible. Our earlier analysis revealing increasing periventricular white matter atrophy with age at the time of original MRI would have been more sensitive to such long term change. ${ }^{14}$ Another plausible scenario is that there is indeed fairly rapid cortical and white matter atrophy, but that it is more widespread and more variable in location compared with the relatively concentrated striatal loss. If this were the case, such atrophy would be harder to detect with any volumetric approach. Such a view is supported by a recent

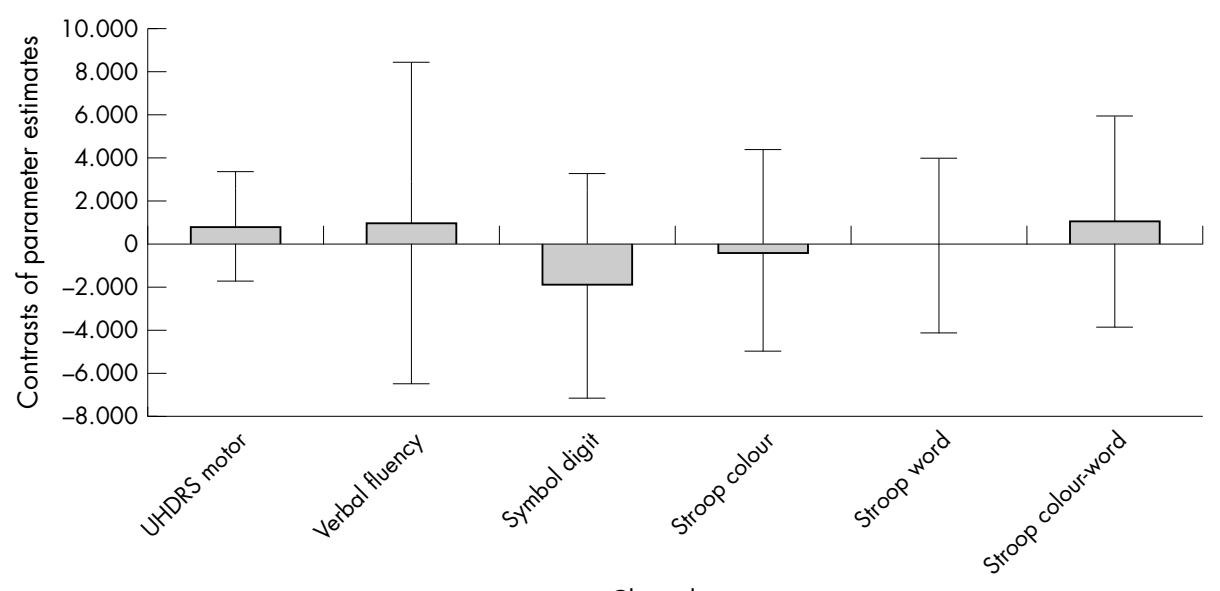

Clinical test

Figure 3 Differences in score over time attributable to the HD genotype for the UHDRS motor and five cognitive tests controlled for the confounding effects of age and education. Contrasts of parameter estimates for mutation-positive minus mutation-negative conditions are plotted with $95 \%$ confidence intervals. Deterioration specific to mutation-positive subjects would result in an increasing motor score and decreasing cognitive scores. 
cross sectional VBM study that demonstrated limited regional cortical atrophy in early HD despite marked striatal involvement. ${ }^{18}$

Progression of disease could not be documented on clinical assessment in this study. This was despite significant, albeit minor, differences in selected motor and cognitive subscores at both initial and follow up assessments. The residual variance in clinical scores not attributable to HD gene status is large, and inevitably larger when different clinicians are responsible for the early and late assessments.

Although it is perhaps not surprising that clinical instruments effective in established HD are insensitive to pathologic progression in preclinical HD, it is indeed remarkable that statistical imaging characterises the distribution and extent of progression in this group with such clarity. We have shown progression of basal ganglia atrophy in HD with an objective, reproducible technique, over a time interval suitable for a trial of intervention, in a mutation-positive preclinical population most likely to receive benefit.

\section{ACKNOWLEDGEMENTS}

Our thanks to Ms R Hatton and Mr D Skerrett for their assistance with image processing, and the Westmead MRI department. We could not have performed this study without the enthusiastic involvement of the volunteers, and Ms J Griffith who helped coordinate the study.

\section{ELECTRONIC-DATABASE INFORMATION}

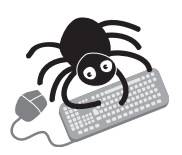

SPM software is available from The Wellcome Department of Imaging Neuroscience at http:// www.fil.ion.ucl.ac.uk/spm. The supplemental material is available from http://jnnp.bmijournals.com/ supplemental/.

\section{Authors' affiliations \\ C M Kipps, A J Duggins, N Mahant, E A McCusker, Department of Neurology, Westmead Hospital, Westmead, NSW 2145, Australia L Gomes, Department of Radiology, Westmead Hospital, Westmead, NSW 2145, Australia}

J Ashburner, Wellcome Department of Imaging Neuroscience, Institute of Neurology, Queen Square, London WCIN 3BG, UK

This study was funded by the Australian Huntington's Disease Association (NSW), and Dr C Kipps was supported by the Ainsworth Movement Disorder Fellowship.

Competing interests: none declared

\section{REFERENCES}

1 DeKosky ST, Marek K. Looking backward to move forward: early detection of neurodegenerative disorders. Science 2003:302:830-4

2 Kremer B. Clinical neurology of Huntington's disease. In: Bates G, Harper P, Jones L, eds. Huntington's disease. Oxford: Oxford University Press, 2002:28-61.

3 A novel gene containing a trinucleotide repeat that is expanded and unstable on Huntington's disease chromosomes. The Huntington's Disease Collaborative Research Group. Cell 1993;72:971-83.

4 Gonzalez-Gonzalez MC, Trujillo MJ, Rodriguez DA, et al. Huntington diseaseunaffected fetus diagnosed from maternal plasma using QF-PCR. Prenat Diagn 2003:23:232-4.

5 Guidelines for the molecular genetics predictive test in Huntington's disease. International Huntington Association (IHA) and the World Federation of Neurology (WFN) Research Group on Huntington's Chorea. Neurology 1994:44:1533-6.

6 McCusker E, Richards F, Sillence D, et al. Huntington's disease: neurological assessment of potential gene carriers presenting for predictive DNA testing. $J$ Clin Neurosci 2000;7:38-41.

7 Kirkwood SC, Siemers E, Bond C, et al. Confirmation of subtle motor changes among presymptomatic carriers of the Huntington disease gene. Arch Neurol 2000;57:1040-4.

8 Kirkwood SC, Siemers E, Stout JC, et al. Longitudinal cognitive and motor changes among presymptomatic Huntington disease gene carriers. Arch Neurol 1999;56:563-8.
9 Smith MA, Brandt J, Shadmehr R. Motor disorder in Huntington's disease begins as a dysfunction in error feedback control. Nature 2000;403:544-9. 10 Rothwell JC. Huntington's disease. A predictor of pathology. Nature 2000:403:495-6.

11 Lawrence AD, Hodges JR, Rosser $A E$, et al. Evidence for specific cognitive deficits in preclinical Huntington's disease. Brain 1998;121:1329-41.

12 Albin RL, Reiner A, Anderson KD, et al. Preferential loss of striato-external pallidal projection neurons in presymptomatic Huntington's disease. Ann Neurol 1992;31:425-30.

13 Gomez-Tortosa E, MacDonald ME, Friend JC, et al. Quantitative neuropathological changes in presymptomatic Huntington's disease. Ann Neurol 2001:49:29-34.

14 Thieben MJ, Duggins AJ, Good CD, et al. The distribution of structural neuropathology in pre-clinical Huntington's disease. Brain 2002; 125:1815-28.

15 Dedeoglu A, Kubilus JK, Yang L, et al. Creatine therapy provides neuroprotection after onset of clinical symptoms in Huntington's disease transgenic mice. J Neurochem 2003;85:1359-67.

16 Chen M, Ona C, Li M, et al. Minocycline inhibits caspase-1 and caspase-2 expression and delays mortality in a transgenic mouse model of Huntington disease. Nat Med 2000;6:797-801.

17 Kieburtz K. A randomized, placebo-controlled trial of coenzyme Q10 and remacemide in Huntington's disease. Neurology 2001;57:397-404.

18 Kassubek J, Juengling FD, Kioschies T, et al. Topography of cerebral atrophy in early Huntington's disease: a voxel based morphometric MRI study. J Neurol Neurosurg Psychiatry 2004;75:213-20.

19 Vonsattel JP, Myers RH, Stevens TJ, et al. Neuropathological classification of Huntington's disease. J Neuropathol Exp Neurol 1985;44:559-77.

20 Lange $\mathbf{H}$, Thorner $G$, Hopf A, et al. Morphometric studies of the neuropathological changes in choreatic diseases. J Neurol Sci 1976;28:401-25

21 Reiner A, Albin RL, Anderson KD, et al. Differential loss of striatal projection neurons in Huntington disease. Proc Natl Acad Sci U S A 1988;85:5733-7

22 Mann DM, Oliver R, Snowden JS. The topographic distribution of brain atrophy in Huntington's disease and progressive supranuclear palsy. Acta Neuropathol (Berl) 1993;85:553-9.

23 Halliday GM, McRitchie DA, Macdonald V, et al. Regional specificity of brain atrophy in Huntington's disease. Exp Neurol 1998;154:663-72.

24 Roos RA, Pruyt JF, de Vries J, et al. Neuronal distribution in the putamen in Huntington's disease. J Neurol Neurosurg Psychiatry 1985;48:422-5.

25 Graveland GA, Williams RS, DiFiglia M. Evidence for degenerative and regenerative changes in neostriatal spiny neurons in Huntington's disease. Science 1985;227:770-3.

26 de la Monte SM, Vonsattel JP, Richardson EP Jr. Morphometric demonstration of atrophic changes in the cerebral cortex, white matter, and neostriatum in Huntington's disease. J Neuropathol Exp Neurol 1988;47:516-25.

27 Aylward EH, Anderson NB, Bylsma FW, et al. Frontal lobe volume in patients with Huntington's disease. Neurology 1998;50:252-8.

28 Harris GJ, Codori AM, Lewis RF, et al. Reduced basal ganglia blood flow and volume in pre-symptomatic, gene-tested persons at-risk for Huntington's disease. Brain 1999;122:1667-78.

29 Aylward EH, Codori AM, Barta PE, et al. Basal ganglia volume and proximity to onset in presymptomatic Huntington disease. Arch Neurol 1996:53:1293-6.

30 Aylward EH, Codori AM, Rosenblatt A, et al. Rate of caudate atrophy in presymptomatic and symptomatic stages of Huntington's disease. Mov Disord 2000;15:552-60

31 Grafton ST, Mazziotta JC, Pahl J, et al. Serial changes of cerebral glucose metabolism and caudate size in persons at risk for Huntington's disease. Arch Neurol 1992;49:1161-7.

32 Aylward EH, Sparks BF, Field KM, et al. Onset and rate of striatal atrophy in preclinical Huntington disease. Neurology 2004;63:66-72.

33 Huntington Study Group. Unified Huntington's Disease Rating Scale: reliability and consistency. Mov Disord 1996;11:136-42.

34 Ashburner J, Andersson JL, Friston KJ. Image registration using a symmetric prior-in three dimensions. Hum Brain Mapp 2000:9:212-25.

35 Freeborough PA, Fox NC. Modeling brain deformations in Alzheimer disease by fluid registration of serial 3D MR images. J Comput Assist Tomogr 1998;22:838-43

36 Ashburner J, Friston KJ. Voxel-based morphometry-the methods. Neuroimage 2000;11:805-21.

37 Hedreen JC, Folstein SE. Early loss of neostriatal striosome neurons in Huntington's disease. J Neuropathol Exp Neurol 1995;54:105-20.

38 Albin RL. Selective neurodegeneration in Huntington's disease. Ann Neurol 1995;38:835-6.

39 Aylward EH, Brandt J, Codori AM, et al. Reduced basal ganglia volume associated with the gene for Huntington's disease in asymptomatic at-risk persons. Neurology 1994;44:823-8.

40 Lasker AG, Zee DS. Ocular motor abnormalities in Huntington's disease. Vision Res 1997;37:3639-45.

41 Rosas HD, Liu AK, Hersch S, et al. Regional and progressive thinning of the cortical ribbon in Huntington's disease. Neurology 2002;58:695-701.

42 Jenkins BG, Rosas HD, Chen YC, et al. IH NMR spectroscopy studies of Huntington's disease: correlations with CAG repeat numbers. Neurology 1998:50:1357-65 